\title{
Two B-type ATP-binding cassette (ABC) transporters localize to the plasma membrane in Thalictrum minus
}

\author{
Nobukazu Shitan ${ }^{1}$, Kazuyoshi Terasaka², Hirobumi Yamamoto ${ }^{3}$, Fumihiko Sato ${ }^{4}$, \\ Kazufumi Yazaki ${ }^{5, *}$ \\ ${ }^{1}$ Department of Natural Medicinal Chemistry, Kobe Pharmaceutical University, Motoyamakita-machi, Higashinada-ku, \\ Kobe, Hyogo 658-8558, Japan; ${ }^{2}$ Department of Pharmacognosy, Graduate School of Pharmaceutical Sciences, Nagoya City \\ University, 3-1 Tanabe-dori, Mizuho-ku, Nagoya, Aichi 467-8603, Japan; ${ }^{3}$ Faculty of Life Science, Toyo University, 1-1-1 Itakura, \\ Gunma 374-0193, Japan; ${ }^{4}$ Graduate School of Biostudies, Kyoto University, Kitashirakawa, Kyoto, Kyoto 606-8502, Japan; \\ ${ }^{5}$ Laboratory of Plant Gene Expression, Research Institute for Sustainable Humanosphere, Kyoto University, Gokasho, Uji, \\ Kyoto 611-0011, Japan \\ *E-mail: yazaki@rish.kyoto-u.ac.jp Tel: +81-774-38-3617 Fax:+81-774-38-3623
}

Received March 11, 2015; accepted June 4, 2015 (Edited by M. Mizutani)

\begin{abstract}
Alkaloids play important roles in plant defenses against herbivores and some alkaloids have medicinal uses. Medicinal alkaloids can be purified from plant tissues or produced axenically in cell culture systems. In culture, cells generally accumulate these toxic metabolites in the vacuole; however, treatment with benzyladenine (BA) induces cultured Thalictrum minus cells to produce the isoquinoline alkaloid berberine, which they release into the medium. A previous biochemical analysis suggested that B-type ATP-binding cassette (ABC) transporters participate in berberine efflux from cultured T. minus cells. In this study, we isolated full-length cDNAs of two novel B-type ABC transporter genes from T. minus, Tmabcb1 and Tmabcb2. The encoded transporters show significant amino acid sequence identity to the Coptis japonica berberine transporters $\mathrm{Cj} \mathrm{ABCB} 1$ and $\mathrm{Cj} \mathrm{ABCB} 2$. Real-time quantitative reverse transcription PCR analyses showed that BA induces an increase in Tmabcb1 and Tmabcb2 mRNA levels in cultured cells. Membrane separation and immunoblot analyses indicated that these proteins localize to the plasma membrane in T. minus cells. These data suggest that TmABCB1 and TmABCB2 participate in berberine transport in T. minus cells.
\end{abstract}

Key words: ATP-binding cassette transporter, berberine, Thalictrum minus.

Plants produce a wide array of secondary metabolites that enable them to adapt to their environment (Croteau et al. 2000). These metabolites include alkaloids, which have diverse chemical structures and various biological activities. Some alkaloids have medicinal applications; for example, morphine is used as an analgesic and vinblastine as an anticancer drug. Stable production of these metabolites in plants requires both biosynthetic enzymes to catalyze metabolic reactions and transport proteins to move end products or biosynthetic intermediates, at the organelle, tissue, and organ levels (Shitan and Yazaki 2008; Shitan et al. 2014). Alkaloids generally accumulate to high levels in a specific organ in intact plants, e.g., strychnine in seeds of Strychnos nuxvomica, nicotine in leaves of tobacco, and berberine in bark of Phellodendron amurense. At the cellular level, sequestration into vacuoles or efflux to the apoplast separates alkaloids from the cytosol and nucleus, possibly providing a species-specific detoxification mechanism for plant cells. However, our knowledge of the membrane transport system underlying alkaloid transport remains limited.

To investigate the mechanism of alkaloid transport, we use cultured Coptis japonica and Thalictrum minus cells as models. Both cell types produce berberine, a benzylisoquinoline alkaloid that has antibacterial activity. C. japonica cells exclusively accumulate endogenous berberine in their vacuoles, and can also absorb exogenous berberine added to the medium (Sato et al. 1990; Sato et al. 1994). By contrast, T. minus cells produce berberine in response to treatment with benzyladenine (BA), and excrete the berberine into the medium (Terasaka et al. 2003a). Even in the absence of BA, T. minus cells can exclude exogenous berberine added to the medium (Terasaka et al. 2003a).

Plant ATP-binding cassette $(\mathrm{ABC})$ transporters move various substrates, using energy obtained by ATP hydrolysis, and are involved in several physiological functions, including detoxification of xenobiotics and transport of hormones and secondary metabolites 
(Kang et al. 2011; Shoji 2014; Verrier et al. 2008; Yazaki et al. 2009). We previously demonstrated that ABC transporters function in berberine uptake by $C$. japonica cells (Sakai et al. 2002) and identified three B-type $\mathrm{ABC}$ transporters, CjABCB1/CjMDR1, CjABCB2, and CjABCB3. We found that $\mathrm{Cj} A B C B 1$ and $\mathrm{Cj} A B C B 2$ are preferentially expressed in the rhizome, where berberine accumulates to high levels in intact $C$. japonica plants, and are responsible for berberine uptake at the plasma membrane to facilitate berberine translocation from the root to the rhizome (Shitan et al. 2003, 2013).

Our previous biochemical studies using transport inhibitors and T. minus cells suggested that B-type ABC transporters mediate berberine efflux, and we identified partial sequences of putative $\mathrm{ABC}$ transporter genes (Terasaka et al. 2003a, 2003b). Here, we attempted to isolate full-length sequences of $\mathrm{B}$-type $\mathrm{ABC}$ transporter genes and characterize the encoded proteins.

To obtain full-length cDNA sequences, we prepared total RNA from T. minus cells from the line Tm103, established in a previous study (Terasaka et al. 2003a), and subsequently performed $5^{\prime}$ and $3^{\prime}$ RACE using the FirstChoice RLM-RACE Kit (Invitrogen). We isolated two full-length cDNAs encoding B-type $\mathrm{ABC}$ transporters that we designated Tmabcb1 (Thalictrum minus abcb1) (Accession no. LC029926) and Tmabcb2 (Accession no. LC029927). The Tmabcb1 and Tmabcb2 cDNAs are each ca. $4.2 \mathrm{~kb}$ long and encode putative polypeptides of 1285 and 1286 amino acids, respectively. A program for predicting transmembrane domains (http://www.cbs.dtu.dk/services/TMHMM/) suggested the presence of 12 transmembrane alpha helices in both TmABCB1 and TmABCB2, similar to human ABCB1 (Ishigami et al. 2013). TmABCB1 and TmABCB2 also each have two predicted nucleotide-binding domains, which form the typical structure of a full-size B-type $\mathrm{ABC}$ transporter.

TmABCB1 and TmABCB2 showed 77\% amino acid sequence identity. In the phylogenetic relationship of plant B-type $\mathrm{ABC}$ transporters, TmABCB1 and 2 belong to the same clade, termed Clade II (Geisler and Murphy 2006) (Figure 1). TmABCB1 showed high amino acid sequence identities to the berberine influx transporters CjABCB1 (78\%) and CjABCB2 (75\%), the auxin influx transporter AtABCB4 (70\%) (Cho et al. 2007; Geisler et al. 2005; Terasaka et al. 2005), and the facultative auxin importer/exporter AtABCB21 (70\%) (Kamimoto et al. 2012). Whereas, TmABCB2 also showed high identity to CjABCB1 (82\%), СјABCB2 (80\%), AtABCB4 (71\%), and AtABCB21 (71\%). Both TmABCB1 and TmABCB2 showed relatively low amino acid sequence identities to Clade I B-type $A B C$ transporters, i.e., the auxin efflux transporters AtABCB1 and AtABCB19 (ca. 44\%) (Yang and Murphy 2009), and the malate importer AtABCB14 (ca. 40\%) (Lee et al. 2008).

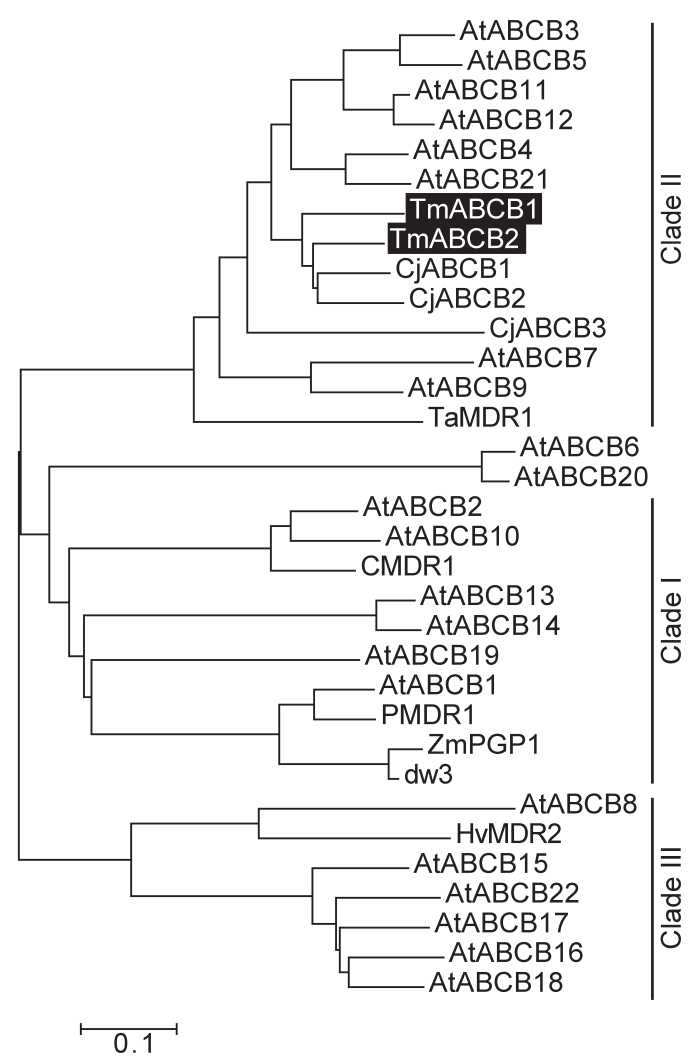

Figure 1. Phylogenetic tree of plant B-type ABC transporters. Fulllength amino acid sequences of plant B-type $A B C$ transporters from Arabidopsis, wheat, barley, potato, upland cotton, C. japonica, and T. minus, were aligned with ClustalW and subjected to phylogenetic analysis using MEGA6 (Tamura et al. 2013) with the neighbor-joining algorithm. Arabidopsis ABCB sequences were recovered according to the AGI-Code described by Verrier et al. (2008). Accession numbers BAB85651, BAB62040, BAM11098, BAM11099, AAF23176, AAD10836, CAA71179, AAR00316, and AAR10387, correspond to the transporters, TaMDR1 (wheat, Triticum aestivum), CjABCB1 (Coptis japonica), CjABCB2, CjABCB3, CMDR1 (cotton, Gossypium hirsutum), PMDR1 (potato, Solanum tuberosum), HvMDR2 (barley, Hordeum vulgare), ZmPGP1 (maize, Zea mays), and dw3 (Sorghum bicolor), respectively. TmABCB1 and TmABCB2 are shaded in black. The scale bar shows the number of amino acid substitutions per site.

We then investigated the expression profile of these transporter genes in T. minus cells using real-time quantitative reverse transcription PCR (qRT-PCR). We cultured the cells in the absence or presence of BA, as described previously (Nakagawa et al. 1984). Total RNA was prepared from cells at 9 days after inoculation, and was reverse transcribed using the ReverTra Ace qPCR RT Master Mix with gDNA remover (TOYOBO) according to the manufacturer's instructions. Primers were designed to amplify each gene separately and rRNA (Accession no. AF479172) was used as a control. Primer sequences were as follows: Tmabcb1-fw, ATC AAG CTA ATA AGG TTC TTT TCA TTC A; Tmabcb1-rv, TGC ATT TTC GAC ATA CAC AAT TAC A; Tmabcb2-fw, AAT CTT CAC TTA CCT AAT TTT TGG TGA TG; Tmabcb2-rv, CTG GGA TTT TGT ATT TAA GCT AAA AGA A; rRNA-fw, ACT CTC TTA AGG 
(A) Tmabcb1

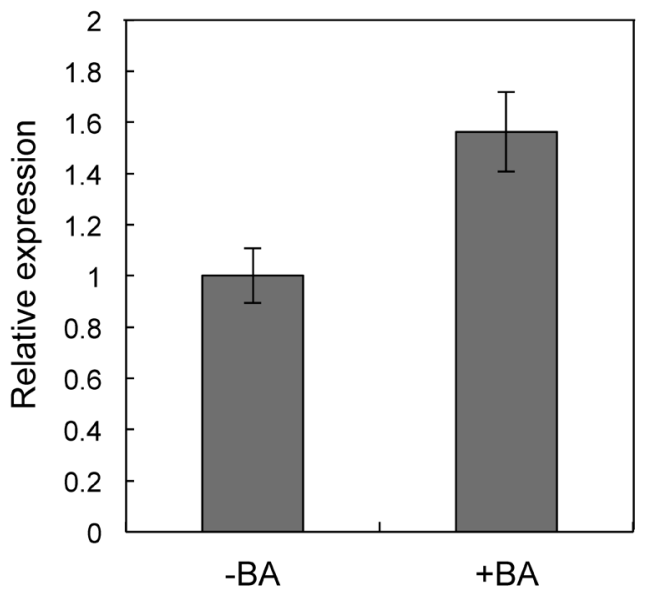

(B) Tmabcb2

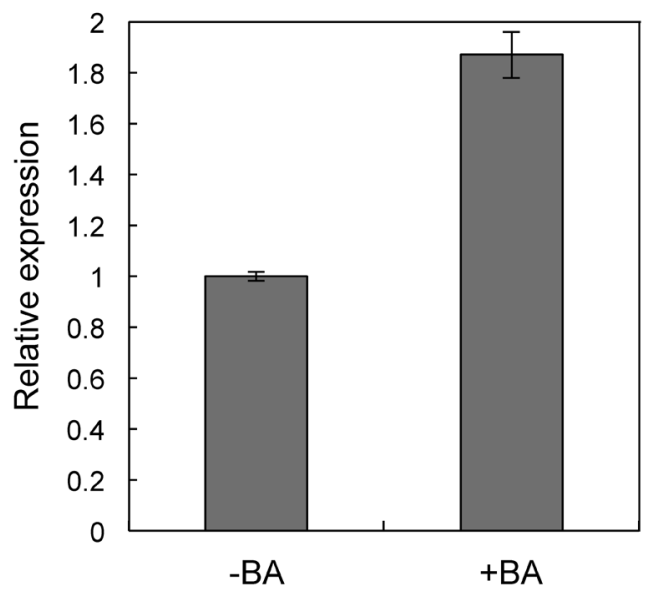

Figure 2. Relative expression of Tmabcb1 (A) and Tmabcb2 (B) in cultured T. minus cells. Expression of each gene was analyzed in cultured T. minus cells for 9 days in the absence $(-)$ or presence $(+)$ of $1 \mu \mathrm{M}$ benzyladenine (BA) by real-time qRT-PCR and rRNA was used as an internal control. Data represent the means \pm SD of three replicates.

TAG CCA AAT G; and rRNA-rv, ATT TCA CAA AGT CGGACT AGA G. PCR was performed in a total volume of $20 \mu \mathrm{l}$, which contained $10 \mu \mathrm{l} 2 \times$ FastStart Essential DNA Green Master (Roche), 10 pmoles of each primer, and cDNA template. The PCR program was as follows: $95^{\circ} \mathrm{C}$ for $10 \mathrm{~min}, 45 \mathrm{cycles}$ of $95^{\circ} \mathrm{C}$ for $10 \mathrm{~s}, 50^{\circ} \mathrm{C}$ for $10 \mathrm{~s}$, and $72^{\circ} \mathrm{C}$ for $25 \mathrm{~s}$. PCR and data acquisition were performed using a LightCycler Nano instrument (Roche). This experiment showed that the transcript levels of both genes increased in response to BA (Figure 2), with Tmabcb2 showing a slightly higher induction ratio than Tmabcb1. Although T. minus cells have berberine efflux activity even in the absence of BA (Terasaka et al. 2003a), the efflux activity might be enhanced in the presence of $\mathrm{BA}$, which induces berberine biosynthesis, to efficiently export endogenous berberine. Therefore, Tmabcb1 and Tmabcb2 may be involved in berberine transport.

We next investigated the subcellular localization of

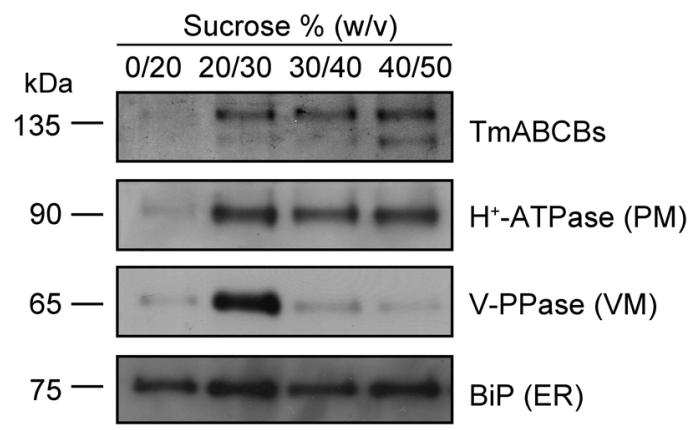

Figure 3. Plasma membrane localization of TmABCBs in T. minus cells. Membrane proteins fractionated by a non-continuous sucrose gradient were separated by SDS-PAGE and immunodetected with antibodies raised against TmABCBs, plasma membrane $\mathrm{H}^{+}$-ATPase, $\mathrm{V}$-PPase, and endoplasmic reticulum BiP.

these transporter proteins. Microsomal membranes from cultured cells were fractionated on sucrose density gradients, subjected to SDS-PAGE, and transferred to an Immobilon polyvinylidene difluoride membrane (Millipore), as described previously (Shitan et al. 2003). Polyclonal antibodies against TmABCBs were raised. A keyhole limpet hemocyanin conjugate of an oligopeptide of a sequence common to both TmABCBs ( $n$-SYPARPDEQIFNGC-c) was injected into a rabbit according to the standard protocol (Iwaki \& Co., Ltd., Tokyo). The membrane was probed with polyclonal antibodies against $\mathrm{Tm} A B C B$ s and with antibodies against several membrane marker proteins. The anti-TmABCB antibodies detected two bands of around $130 \mathrm{kDa}$. The fractionation pattern of TmABCBs resembled that of plasma membrane $\mathrm{H}^{+}$-ATPase and clearly differed from those of the vacuolar $\mathrm{H}^{+}$-pyrophosphatase (V-PPase), a tonoplast marker, and membranes containing a luminal binding protein (BiP), a marker of the endoplasmic reticulum (Figure 3). These results indicate that TmABCB1 and TmABCB2 localize to the plasma membrane and not to the tonoplast or the endoplasmic reticulum.

The Tmabcb1 and Tmabcb2 mRNAs were induced by BA treatment, which also induces berberine biosynthesis. TmABCBs localize to the plasma membrane. These data suggest that TmABCBs mediate berberine efflux at the plasma membrane in T. minus cells. However, the possibility that TmABCBs might function as berberine importer cannot be denied, because both TmABCB1 and TmABCB2 had high amino acid sequence similarities to the berberine influx transporters $\mathrm{Cj} A \mathrm{ABCB} 1$ and CjABCB2, and AtABCB21, which can import or export auxin depending on the condition. This similarity suggests the possibility that TmABCBs might function in influx or efflux of berberine, or perhaps both, similar to AtABCB21. In the rhizome of T. flavum, a similar species of $T$. minus, the presence of berberine importer and exporter was suggested, because berberine probably 
(A)

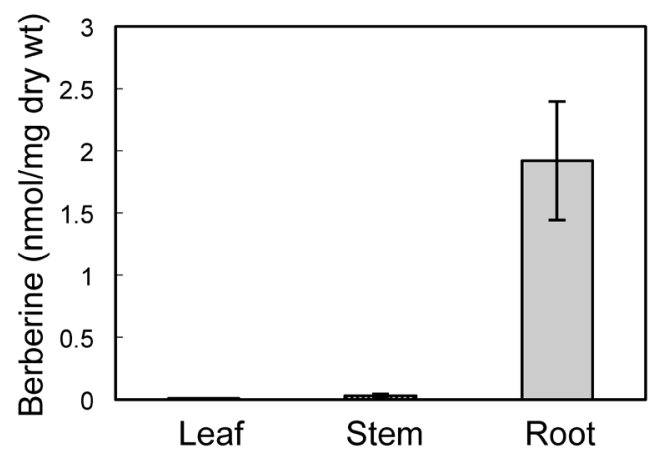

(B) Tmabcb1

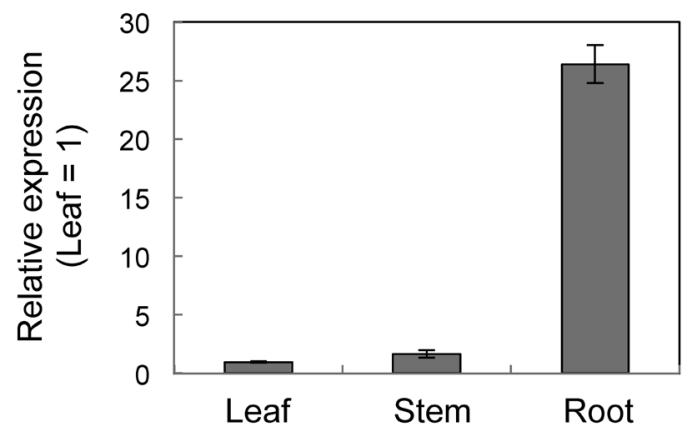

(C) Tmabcb2

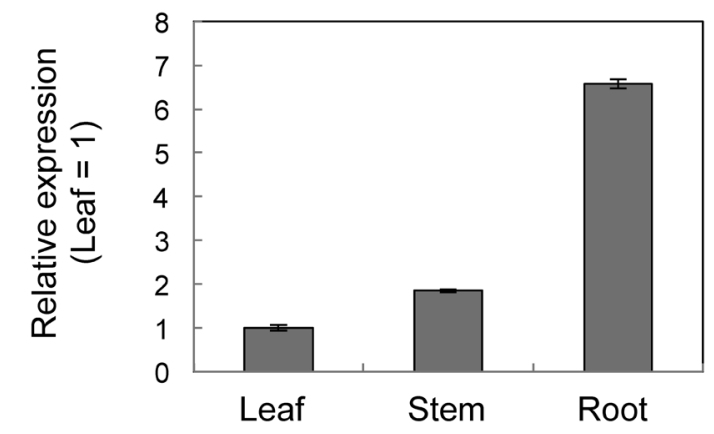

Figure 4. Root-specific accumulation of berberine and expression of Tmabcb1 and Tmabcb2 in intact T. minus plants. (A) From each dried tissue of T. minus, berberine was extracted using $\mathrm{HCl}$-acidified methanol. The methanol extract was subjected to HPLC analysis as described previously (Shitan et al. 2005). (B) Relative expression of Tmabcb1 (B) and Tmabcb2 (C) in intact T. minus plants. Expression of each gene was analyzed by real-time qRT-PCR and rRNA was used as an internal control. Data represent the means \pm SD of three replicates.

moves from the apical meristem, the biosynthesis site, to the pith and cortex, the accumulation sites (Samanani et al. 2005). In intact T. minus plants, although rhizome was not examined due to the small size of analyzed plants, berberine accumulates to high levels in root tissues (Figure 4). Since Tmabcb1 and Tmabcb2 are preferentially expressed in roots (Figure 4), TmABCB1 and TmABCB2 might contribute to retention of berberine by functioning as berberine importer in this tissue. The functions of TmABCB1 and TmABCB2, however, remain to be clarified due to difficulties in subcloning the full-length
cDNAs (ca. $4 \mathrm{~kb}$ ) encoding these proteins into expression vectors, which causes many mutations during subcloning by unknown mechanisms. Further study is required to clarify their transport substrate and physiological functions.

Transport of secondary metabolites has been intensively studied in plants and several transporters have been identified (Nour-Eldin and Halkier 2013; Shitan and Yazaki 2008; Shitan et al. 2014; Zhao and Dixon 2010). Engineering plants with altered transport properties may have applications in the production of valuable compounds (Nour-Eldin and Halkier 2013). The findings presented here improve our understanding of alkaloid transport in T. minus. Identifying and characterizing additional alkaloid transporters, including TmABCBs, will provide further insight into alkaloid transport mechanisms in plants.

\section{Acknowledgements}

We thank Dr. M. Boutry (Université Catholique de Louvain, Belgium) for anti- $\mathrm{H}^{+}$-ATPase antibodies; Dr. N. Koizumi (Osaka Prefecture University, Japan) for anti-BiP antibodies; Dr. M. H. Sato (Kyoto Prefectural University, Japan) for anti-V-PPase antibodies. Analysis of DNA sequencing was conducted with the Life Research Support Center in the Akita Prefectural University. This work was supported by a Grant-in-Aid for Scientific Research 10217206 (to K.Y.) and JSPS Fellows 04J00405 (to N.S.).

\section{References}

Cho M, Lee SH, Cho HT (2007) P-glycoprotein4 displays auxin efflux transporter-like action in Arabidopsis root hair cells and tobacco cells. Plant Cell 19: 3930-3943

Croteau R, Kutchan TM, Lewis NG (2000) Natural Products (Secondary Metabolites). In: Buchanan B, Gruissem W, Jones R (eds) Biochemistry \& Molecular Biology of Plants. American Society of Plant Physiologists, Maryland, pp 1250-1318

Geisler M, Blakeslee JJ, Bouchard R, Lee OR, Vincenzetti V, Bandyopadhyay A, Titapiwatanakun B, Peer WA, Bailly A, Richards EL, et al. (2005) Cellular efflux of auxin catalyzed by the Arabidopsis MDR/PGP transporter AtPGP1. Plant J 44: 179-194

Geisler M, Murphy AS (2006) The ABC of auxin transport: the role of p-glycoproteins in plant development. FEBS Lett 580: 1094-1102

Ishigami M, Tominaga Y, Nagao K, Kimura Y, Matsuo M, Kioka N, Ueda K (2013) ATPase activity of nucleotide binding domains of human MDR3 in the context of MDR1. Biochim Biophys Acta 1831: 683-690

Kamimoto Y, Terasaka K, Hamamoto M, Takanashi K, Fukuda S, Shitan N, Sugiyama A, Suzuki H, Shibata D, Wang B, et al. (2012) Arabidopsis ABCB21 is a facultative auxin importer/exporter regulated by cytoplasmic auxin concentration. Plant Cell Physiol 53: 2090-2100

Kang J, Park J, Choi H, Burla B, Kretzschmar T, Lee Y, Martinoia E (2011) Plant ABC Transporters. The Arabidopsis book/American Society of Plant Biologists 9: e0153

Lee M, Choi Y, Burla B, Kim YY, Jeon B, Maeshima M, Yoo JY, Martinoia E, Lee $\mathrm{Y}$ (2008) The ABC transporter AtABCB14 is a malate importer and modulates stomatal response to $\mathrm{CO}_{2}$. Nat 
Cell Biol 10: 1217-1223

Nakagawa K, Konagai A, Fukui H, Tabata M (1984) Release and crystallization of berberine in the liquid medium of Thalictrum minus cell suspension cultures. Plant Cell Rep 3: 254-257

Nour-Eldin HH, Halkier BA (2013) The emerging field of transport engineering of plant specialized metabolites. Curr Opin Biotechnol 24: 263-270

Sakai K, Shitan N, Sato F, Ueda K, Yazaki K (2002) Characterization of berberine transport into Coptis japonica cells and the involvement of ABC protein. J Exp Bot 53: 1879-1886

Samanani N, Park SU, Facchini PJ (2005) Cell type-specific localization of transcripts encoding nine consecutive enzymes involved in protoberberine alkaloid biosynthesis. Plant Cell 17: 915-926

Sato F, Takeshita N, Fujiwara H, Katagiri Y, Huan L, Yamada Y (1994) Characterization of Coptis japonica cells with different alkaloid productivities. Plant Cell Tissue Organ Cult 38: 249-256

Sato H, Kobayashi Y, Fukui H, Tabata M (1990) Specific differences in tolerance to exogenous berberine among plant cell cultures. Plant Cell Rep 9: 133-136

Shitan N, Bazin I, Dan K, Obata K, Kigawa K, Ueda K, Sato F, Forestier C, Yazaki K (2003) Involvement of CjMDR1, a plant multidrug-resistance-type ATP-binding cassette protein, in alkaloid transport in Coptis japonica. Proc Natl Acad Sci USA 100: $751-756$

Shitan N, Dalmas F, Dan K, Kato N, Ueda K, Sato F, Forestier C, Yazaki K (2013) Characterization of Coptis japonica CjABCB2, an ATP-binding cassette protein involved in alkaloid transport. Phytochemistry 91: 109-116

Shitan N, Kato K, Shoji T (2014) Alkaloid transporters in plants. Plant Biotechnol 31: 453-463

Shitan N, Kiuchi F, Sato F, Yazaki K, Yoshimatsu K (2005) Establishment of Rhizobium-mediated transformation of Coptis japonica and molecular analyses of transgenic plants. Plant Biotechnol 22: 113-118
Shitan N, Yazaki K (2008) Membrane transport of plant secondary metabolites. In: Jaiwal PK, Singh RP, Dhankher OP (eds) Plant Genetic Engineering volume 9: Plant Membrane and Vacuolar Transporters, pp 283-300

Shoji T (2014) ATP-binding cassette and multidrug and toxic compound extrusion transporters in plants: a common theme among diverse detoxification mechanisms. Int Rev Cell Mol Biol 309: 303-346

Tamura K, Stecher G, Peterson D, Filipski A, Kumar S (2013) MEGA6: Molecular Evolutionary Genetics Analysis version 6.0. Mol Biol Evol 30: 2725-2729

Terasaka K, Blakeslee JJ, Titapiwatanakun B, Peer WA, Bandyopadhyay A, Makam SN, Lee OR, Richards EL, Murphy AS, Sato F, et al. (2005) PGP4, an ATP binding cassette P-glycoprotein, catalyzes auxin transport in Arabidopsis thaliana roots. Plant Cell 17: 2922-2939

Terasaka K, Sakai K, Sato F, Yamamoto H, Yazaki K (2003a) Thalictrum minus cell cultures and ABC-like transporter. Phytochemistry 62: 483-489

Terasaka K, Shitan N, Sato F, Maniwa F, Ueda K, Yazaki K (2003b) Application of vanadate-induced nucleotide trapping to plant cells for detection of ABC proteins. Plant Cell Physiol 44: 198-200

Verrier PJ, Bird D, Burla B, Dassa E, Forestier C, Geisler M, Klein M, Kolukisaoglu U, Lee Y, Martinoia E, et al. (2008) Plant ABC proteins: a unified nomenclature and updated inventory. Trends Plant Sci 13: 151-159

Yang H, Murphy AS (2009) Functional expression and characterization of Arabidopsis ABCB, AUX 1 and PIN auxin transporters in Schizosaccharomyces pombe. Plant J 59: 179-191

Yazaki K, Shitan N, Sugiyama A, Takanashi K (2009) Cell and molecular biology of ATP-binding cassette proteins in plants. Int Rev Cell Mol Biol 276: 263-299

Zhao J, Dixon RA (2010) 'ins' and 'outs' of flavonoid transport. Trends Plant Sci 15: 72-80 